DE DE GRUYTER

OPEN

G

BULGARIAN ACADEMY OF SCIENCES

CYBERNETICS AND INFORMATION TECHNOLOGIES • Volume 15, No 6

Special Issue on Logistics, Informatics and Service Science

Sofia • 2015

Print ISSN: 1311-9702; Online ISSN: 1314-4081

DOI: 10.1515/cait-2015-0080

\title{
Visualization Emergency Research Based on Mobile Mapping Technology
}

Liao Ming ${ }^{1,2,3}$, Pan Yuan Fang ${ }^{2,4}$

${ }^{1}$ School of Remote Sensing and Information Engineering, Wuhan University, 430079 China

${ }^{2}$ Key Laboratory of Watershed Ecology and Geographical Environment Monitoring, National Administration of Surveying, Mapping and Geoinformation, 2166 Fanghu Road, Nanchang, 330209 China

3 Jiangxi Provincial Geomatics Center , 2166 Fanghu Road, Nanchang, 330209 China

4 Jiangxi Provincial Geographical National Conditions Monitoring and Remote Sensing Institute, 2166 Fanghu Road, Nanchang, 330209 China

Email: c9803244@gmail.com

Abstract: Considering the prevention and management of the major emergencies in China and the increasingly severe environmental security situations, a visualization emergency solution, based on a mobile mapping system is proposed. This paper studies the key technologies of a mobile mapping system in emergency management. It considers the design of a total factor and full-texture real map, which is measurable and can be marked through the mobile mapping technology. It also proposes the application of a real map in emergency cases, which has large practical significance.

Keywords: Mobile mapping, visualization, emergency.

\section{Introduction}

With the development of economic prosperity, the prevention and management of major sudden emergencies has become a vital political task for different nations and regional governments in the world. The public security system facilities, including emergency aid, security defence, anti-terrorism facilities, need to be constructed. With the rapid development of Chinese economic globalization, China has and will be widely involved in the global economic environment, which will significantly increase the risk of major public safety incidents in the urban area. One isolated 
incident can produce a diffusion effect and become a huge social disaster, which poses great threat on the national security, social stability and people's normal life. For this reason, the city public safety emergency command system has emerged.

In recent years China has devoted a lot to the information construction of an emergency command. However, with the rapid development of national economy, the city construction changes with each passing day, urban population and building density keeps swelling, the height of tall buildings increases constantly, the number of public places, such as shopping malls, Internet bars, entertainment centers increases dramatically. On one hand, it illustrates the increasing difficulties of an emergency command. On the other hand, it shows that the information management of the emergency command is behind time. There are several main problems.

The geographic data is not comprehensive and cannot meet the demands of the emergency command and rescue. More than ninety percents of the operational data needed in the emergency command, are related to the geographic location data. The emergency command geographic information system is needed to provide the decision making support, whether it is for a quick response of the rescue, location of the police resources, selection of paths, enquiry of buildings, or for better knowledge of the safety passages in crowded places and storage of dangerous goods. However, the core of GIS is the geographic information data. Without the support of comprehensive, accurate and real-time data, GIS system could not play its role in the decision making support. In other words, no matter how advanced the communication apparatus and computer networks are, the whole emergency command system would not be able to operate at its full potential without complete geographic data.

The real time update of the data is lagging behind. In addition, the information contained in an emergency command GIS should be kept updated to correctly express the reality of the city. City construction changes each day. For a megalopolis like Beijing, if the map is not updated within one year, over 40 percent of the data will become invalid; the map will become a waste one if not updated within two years, let alone the operational data needed by an emergency command. Therefore, in general the emergency command geographic information must be updated at least three times a year. For centers of commercial distribution and floating population and so on, the update cycle should be even shorter to meet the demand. Consequently, the emergency command departments are in urgent need for a complete data solution that could meet the demands and establish a long-term data update mechanism.

The low efficiency hardly meets the map update requirements. Under emergency conditions, the key government departments need to make quick, accurate and efficient decisions based on the latest information and circumstances. As time goes on, different institutions have developed their own databases for different purposes. The growing capacity of the database and the efficient utilization of the data is a huge challenge faced by the key departments. The expensive data source has an important value and significance to relevant command and decision making of the government. 
Therefore, there is a pressing need for a better GIS data source and better visualization tools to transfer more abundant information. This better data source includes 3D modelling, real 3D, virtue 3D, etc. The stereoscopic sensation and immersion experience of 3D modeling is undoubtedly stronger than that of real 3D. However, the production of 3D modeling needs a lot of manpower and material resources, especially when high authenticity is required. Since the shooting and making of real 3D is relatively simple and convenient, and the production efficiency is very high, the real 3D could not only keep a full record of the scene somewhere in time, but also display the scenes in a three-dimensional way. This cost-efficient display mode and record tools cannot be accomplished by any other general picture representation or 3D modelling.

During the Operation Iraq Freedom period, a new discipline, called GEOspatial INTelligence (GEOINT) gives the USA a decisive advantage of technology. GEOINT helps the soldiers at a platoon level to understand better the Earth in an unprecedented way and finally "points to the road to Baghdad" [1]. In fact, the first necessity of grasping geospatial intelligence is the image. People could absorb large amounts of information at a rapid speed through visualization. GEOINT could provide visual description and dynamic comprehension of the geographic conditions. Visualization, an indispensable guarantee, could help the war-fighters grasp the information quickly and deal with high-traffic information quickly. As an old saying goes: "a picture is worth a thousand words". Virtual image is a very good medium to help fill the gaps of deficient information of GIS visualization. There are also a lot of GEOINT applications in industries like public security, emergency response, disaster relief, anti-terrorism and infrastructure in the United States.

Well known international enterprises like Google and Microsoft are making researches on Street View technology. They try to obtain detailed information like the road slope, turning circle and lane width via a Mobile Mapping System, hence provide the data basis for public services. However, there is little research on emergency relief. Chinese Internet giants like Baidu, Tencent, Alibaba, Sina, Sohu, Netease are developing their own applications of public services. Traditional twodimensional electronic map is outdated to an emergency command. However, Panoramic Laser System, which could obtain panoramic images and threedimensional point cloud data and generate real three-dimension geographic information, will be the top choice to gain data in emergency management applications in the future [2].

National Geospatial-Intelligence Agency (NGA) deems that only image emergency solution is able to provide leaders with keen insights. Based on the images and conform to human interpretation, a real emergency system has become the main stream in emergency development. Real 3D is with no doubt the best choice when we need to represent scenes authentically, intuitively, and comprehensively for recording or display. It helps all stakeholders to quickly understand the overall and local situation of the scene, thereby work out more efficient preventive security measures and emergency plans, deploy careful stakeout 
actions and improve execution efficiency. A real map with the function of measurement is the first choice.

This paper proposes for the first time to use the image map as the geographic data platform of emergency management, and a unified platform with other police classification and emergency response departments. It also invents a series of methods for data management and distribution, including direct labelling, linkage, inquiry, measurement and scan. For the first time this study applies the world leading mobile road mapping technology to the construction and update of geographic data for fire protection. It introduces the massive data solution of streetscape image database and addresses a long-time bottleneck problem in data management in fire-fighting area.

\section{Visualization emergency research based on mobile mapping technology}

This paper states the importance and significance of city 3D visualization emergency, and proposes its basic technology - mobile mapping. The paper further conducts an in-depth analysis of the mobile mapping technology, which laid the foundation for the application of 3D visualization emergency.

Real maps with mapping functions can be obtained by a mobile mapping system. Using a compact modular design, the mobile mapping system is composed by a data collection device, a monitoring device and a power source, these three subsystems being connected by aviation cables and plugs. The data collection device consists of high-precision optical fibre or laser Inertial Navigation System (INS), Charge-Coupled Device (CCD) camera, LIDAR and high-grade protective cover. The INS, CCD, and LIDAR devices are connected through a fixed precision mechanical device. These well designed mechanical devices can be easily installed on any mobile vehicles, trains, airplanes, ships and other mobile carries, without outdoor calibration. These devices are capable for mapping under any "sea-airland" conditions, which performs data collection functions for visualization emergency.

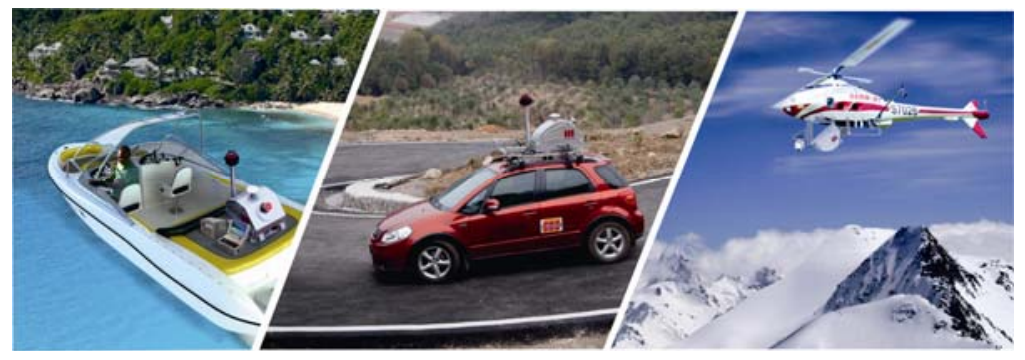

Fig. 1. A mobile mapping system

Digital Measurable Image (DMI) is a processed product from the data collected by using mobile mapping systems. The DMI development kit can support direct mapping on the three-dimensional image, information extraction and 
integration with other basic geographic products, which is a new emergency product developed by Chinese emergency database to adapt to the new requirements of demand-driven data collection and spatial information update. In this way, this tool uses high definition, high resolution images to directly map the original appearance of the object and the environment. It contains the information of the targeted objects being measured, as well as various kinds of natural and social information related to the object. It is the most intuitive carrier of city 3D visualization emergency, its image is the most intuitive and the most realistic portrayal of the objective world.

\section{Mobile mapping technology-based emergency system architecture}

The system consists of three major components: data processing software, data management software and data application platform (Fig. 2).

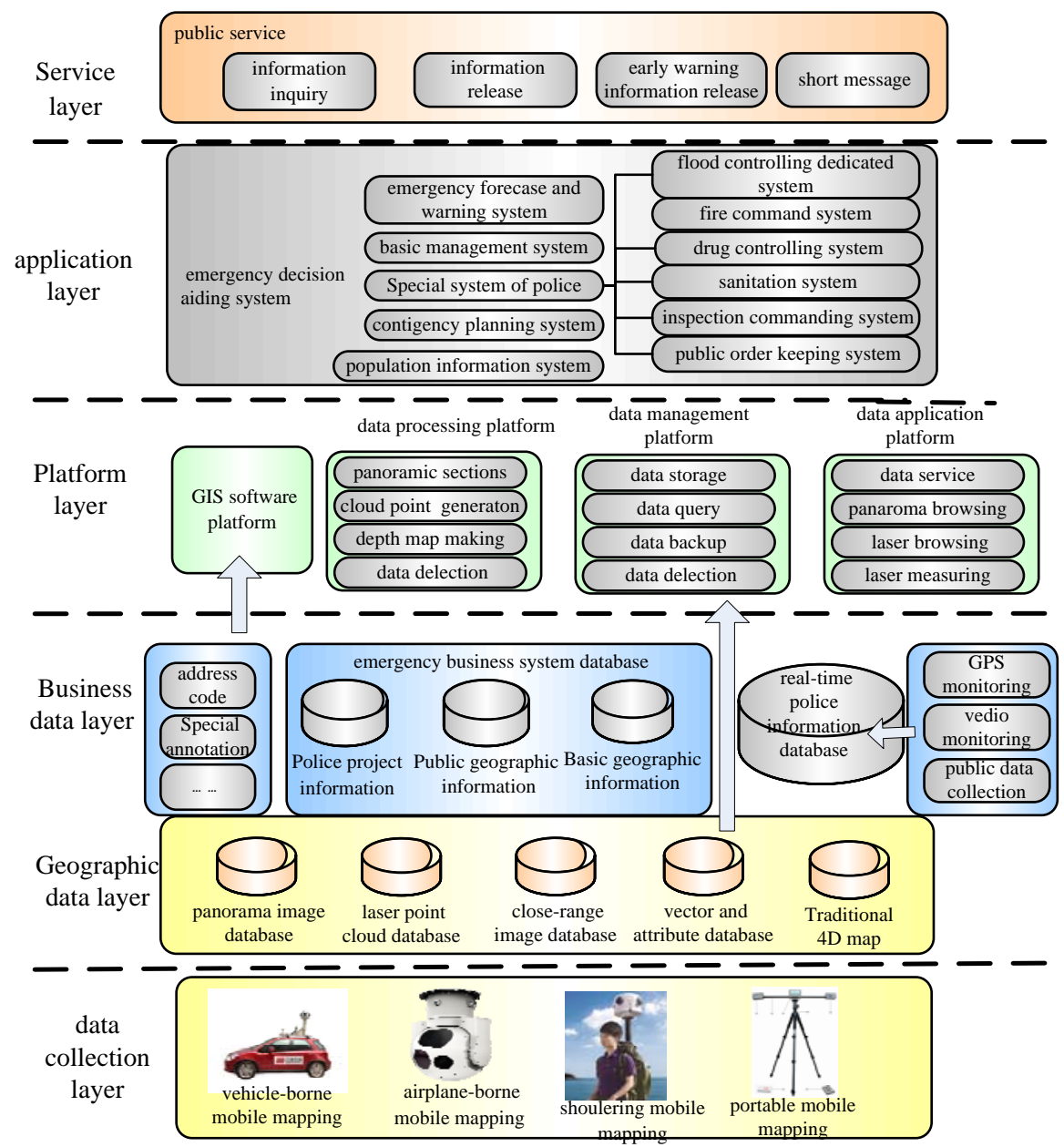

Fig. 2. The system architecture diagram 
Data collection layer. Vehicle-borne mobile mapping, airplane-borne mobile mapping, shouldering mobile mapping, portable mobile mapping.

Geographic data layer. This layer consists of the geographical data collected by the data collection layer and the business data.

Platform layer. The data processing software is used to convert the application format of the collected emergency data, including the conversion and processing of the image data and laser cloud point data; The data management software is used to store and manage the raw emergency data and the converted result data, it provides functions, such as data storage, data query, data backup and data deletion. The data emergency application platform can be used to mark, link, query, measure and browse the result data.

Application layer. This layer can support functions, such as emergency forecast and early warning, response alarm/rescue command, decision support and satellite navigation.

Service layer. Web Server: response to the access from the Web; CTI Server: telephone voice access and distribution; SMS Server: a short message received, sent, decoding; GIS server: the processing of the electronic map image data; Application Server: coordination, communication and collaboration between different services and systems.

\section{The mobile mapping-based city 3D visualization technology}

Mobile mapping, as one of the cutting-edge technologies in today's world of surveying and mapping, is an integrated innovation of various high-tech products. The mobile measurement system includes data collection, data processing and 3D visualization, the technology used is as follows:

\subsection{Multi-sensor integration technology}

Mobile mapping needs to have integrated control of a GNSS, an inertial gyroscope, an accelerometer and other sensors, in order to complete the mapping of the position, attitude and velocity with high frequency and a sync navigation solution as well. On the other hand, camera (visible or infrared), LIDAR, Synthetic Aperture Radar (SAR), gravity meter, environmental measuring instruments and other instruments need to be integrated in the application. The development of a mobile mapping-based system is a systematic project, among which the multi-sensor integration is one of the key techniques.

\subsection{GNSS/Inertial navigation and combination navigation technology}

The mobile mapping system obtains the position and attitude data by using GNSS/IMU, and specifically establishes the IMU static and dynamic error compensation and navigation solution algorithm, thus the system can output the post-compensation space observation information (i.e., angle increment, speed increment), and it is also able to output the observation data posture calculating the results in real time. This technology features a wide temperature range and high 
dynamic response frequency, which can meet the requirements of most of the navigation and control fields.

Based on the successful development of a fibre optic IMU, the tightly integrated algorithm has been recognized as a worldwide data challenge. This technology has made breakthrough in autonomous positioning and orientation hardware combination, as well as the positioning integration algorithm, which solves the direct geo referencing problem of ground remote sensing in complex vehicle-bone environment.

\subsection{High-precision PPOI information integration technology}

Mobile mapping, aerial photogrammetry survey and other fields typically require real time calculation of the position and attitude for real time measurement, control and data quality assurance. From the application requirements of a mobile mapping point of view, a high-precision PPOI system information integration technology, based on the properties of the equipped carrier has been developed, the scientific data processing model and PPOI information integration processing software were developed as well. The main contents include:

1. Establishing of a typical GPS/IMU strap-down integration algorithm, and development of the embedded real time navigation solution software and monitoring software;

2. Applying the enhanced Kalman filter model to improve real-time response capabilities;

3. The precision position and orientation algorithm adopts the post-processed reverse smoothing techniques and reserves the image processing feedback correction interfaces, such as Analytic Aerial Triangulation (AAT) to compensate PPOI system deviations which occur in some scenarios (such as a long cruise), and therefore enhance the accuracy of position and orientation.

\subsection{Massive mobile mapping data visualization processing technology}

Large-scale multi-source data Collaboration Mapping software (CoMapper) is designed for massive emergency mobile mapping data processing. CoMapper is a tool for the integration of data collecting and editing, it can efficiently complete various tasks, including closed-up ground image data collection required by the land-based mobile mapping systems, mapping the laser cloud point data, attribute assignment, editing, mapping, storage and quality control, it can also integrate and process other sources data, such as aerial remote sensing data, vector maps.

\subsection{Service-type live 3D data management and display technology}

The service-type live 3D data management and application platform (TrueMap Server) is an application development platform supporting all types of 3D data management and application service, which has open cloud service capabilities to support a variety of secondary development tools, and to provide PB grade mass real three-dimensional image data (i.e., DMI, continuous panorama, panoramic 
single point, laser, tilt photography, etc.) and clustered data management of map tiles, five advanced features including real image search, real image photo navigation, on-demand mapping, real map management, real image labels.

With a mobile terminal, a desktop terminal and Web terminal integrated product solutions provided, this technology is compatible with all types of GIS platform software and industry applications, which can easily build applications based on the real map for urban management, public security, emergency, transportation, railways, tourism and other government departments management systems and street maps for a public service website.

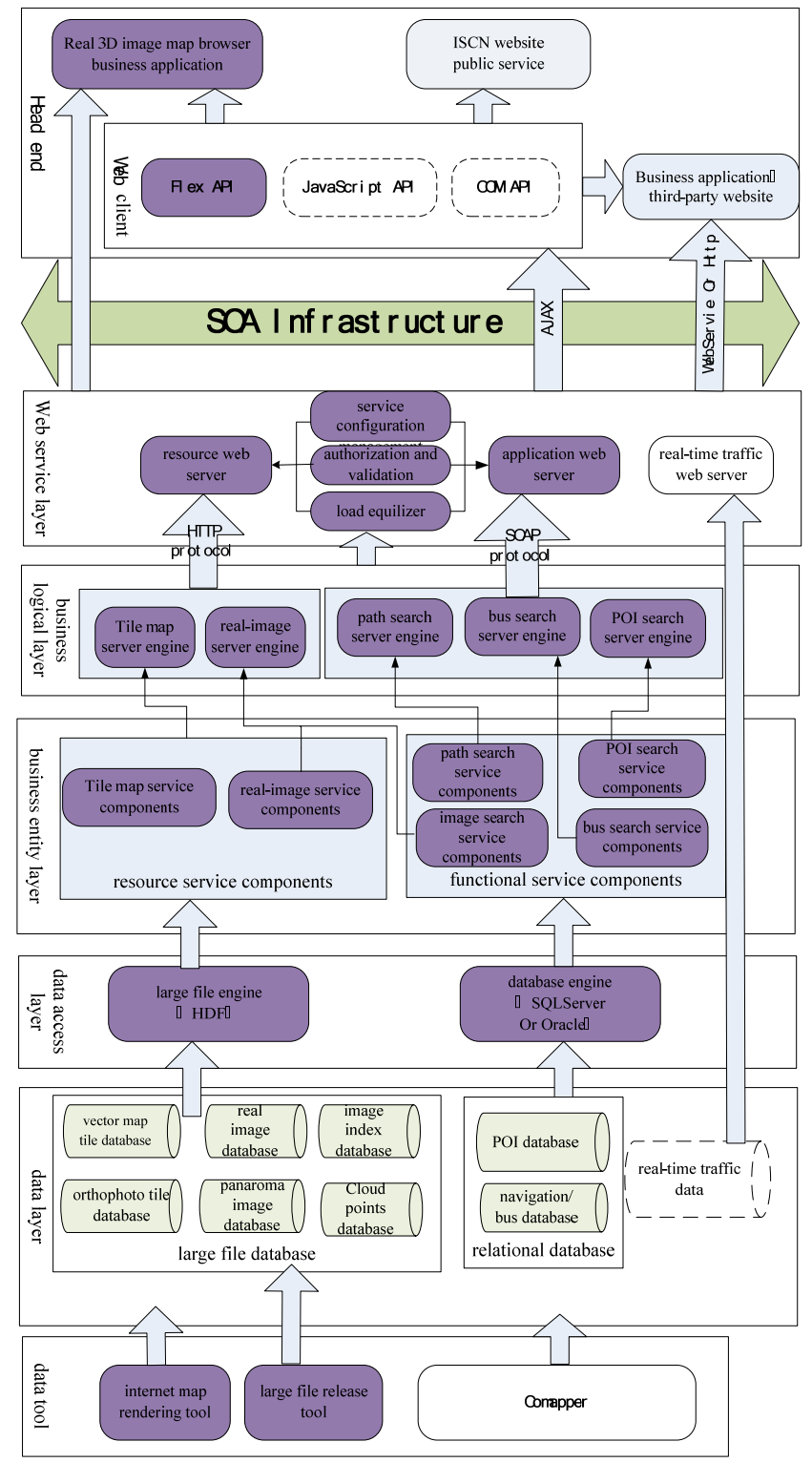

Fig. 3. The platform architecture diagram 
The Web client designed for Web developers consists of a real image component, a map component and other Software Development Kits (SDK) and developer documentation, providing RIA and Web Service these two modes of development. Its upper layer through an open interface integrates with the developer's application, and the lower layer conducts information and data exchange through Ajax technology and a Web server. The Web service layer consists of resource servers, application servers (GIS server), service configuration management components and authorization verification components. The resource server is responsible for parsing Http request from a Web client and assigning the request to the function server, and passing the request results (resources returned from the business layer) back to the Web client. The business logical layer consists of a tile map service engine, real-image service engine, path search service engine, bus search service engine, and POI search service engine. The concept of a service engine is actually regarded as the management capabilities of a number of distributed services, which include service registration, cancellation, status checking, request redirections and service responses forwarding. The business entity layer consists of tile map service components, real-image service components, path search service components, image search service components, POI search service components and six bus search service components. The data access layer consists of a large file engine and database engine. The large file engine retrieves and reads the related resources from the large file (or database), and provides a unified call interface for different platforms or different databases. The data layer is composed of a series of standard data. The lowermost layer consists of the necessary software and tools to support the Truemap Server.

\section{The realization of function modules of a $3 \mathrm{D}$ visualization emergency display system}

\subsection{Emergency real image search/navigation function}

Function: Coordinates the search-based emergency image navigation, rectangle scope search-based emergency image navigation, the emergency image navigation based on the search of the central latitude/longitude and radius, image-based emergency path search, outputs the current position coordinates and direction based on the emergency image

Function design description: Based on the parameter information, location of the key points back and forth (i.e., anchor points) reflected on an image, and crossroad direction signs for quick search of the algorithm and opens the interfaces.

\subsection{Emergency real image browsing function}

Function: Automatically forward and backward playback browsing based on a real image, single-frame forward/backward manually browsing, single large-scale image browsing, image zoom in and zoom out, intersection navigation.

Function design description: Enables the of display the previous frame and a frame after the current real image, and displays the left frame and the right frame 
images of the current real image. Automatically controls the speed of real time video playback, and provides open interfaces.

\subsection{Emergency image labelling/linking function}

Function: Adds/modifies/deletes the labelling and label properties, label search, query labels based on the image name, label recheck image, management of label symbols.

Function design description: Component display, clicking the component layer control, open the component layer, and displays the components in the real image; Component positioning: moving the mouse to the component label when the image switches to the component label, clicking the mouse, the position of the component and its detailed information will display on a two-dimensional map; View the component information: Clicking on the component label on the image, a sheet containing detailed information of the component will pop up.

\subsection{Emergency real image mapping function}

Function: Point measuring, line measuring of the length, width and height.

Function Description: Length measurement: locates the start and end points in a three-dimensional relative, measures the length between the start and end points, and opens the following interfaces:

\begin{tabular}{|c|c|}
\hline Description & Interface \\
\hline \multirow{4}{*}{$\begin{array}{l}\text { 1.Open mapping } \\
\text { interface }\end{array}$} & [Call Interface] openMeasureUI () \\
\hline & [Parameter] N/A \\
\hline & [Return Value] void \\
\hline & $\begin{array}{l}\text { [Description] } \\
\text { Open measuring interface, achieve length, width and height } \\
\text { measurements }\end{array}$ \\
\hline \multirow{4}{*}{$\begin{array}{l}\text { 2. Close mapping } \\
\text { interface }\end{array}$} & [Call Interface] closeMeasureUI() \\
\hline & [Parameter] N/A \\
\hline & [Return Value] void \\
\hline & [Description] Close measuring interface \\
\hline \multirow{4}{*}{$\begin{array}{l}\text { 3. Obtain camera } \\
\text { math and spatial } \\
\text { coordinates }\end{array}$} & $\begin{array}{l}\text { [Call Interface] } \\
\text { measureSeverKit(leftPoi: Point, rightPoi: Point, leftid: String, } \\
\text { rightid: String, url: String) }\end{array}$ \\
\hline & $\begin{array}{l}\text { [Parameter] } \\
\text { leftPoi: the position point(x,y) of the matched image on the left } \\
\text { rightPoi: the position point(x,y) of the matched image on the right } \\
\text { leftid: the imageID (image name) of the image on the left } \\
\text { righted: the imageID (image name) of the image on the right } \\
\text { url: service address requests }\end{array}$ \\
\hline & $\begin{array}{l}\text { [Return Value] } \\
\text { sendMeasureInfo:Callback function to obtain the measurement } \\
\text { results }\end{array}$ \\
\hline & $\begin{array}{l}\text { [Description] } \\
\text { Connect point and line measuring interface cable, obtain the } \\
\text { spatial coordinates of the image }\end{array}$ \\
\hline
\end{tabular}




\section{Design of the 3D visualization emergency scenario}

\subsection{Construction of the visualization database of emergency facilities}

Water, fire hydrants, fire exits, hazardous materials, etc., are the key emergency information that is needed for unified management. The commercial and high population concentration areas are the high-potential areas for an emergency incidence, where various rescue equipment and passage situation requires strict information management, in order to prepare for contingency plans and general queries. The system uses MMS technology to locate small targets, such as fire hydrants and other hazards, while combining the information with the existing data in the building, in order to grasp a comprehensive understanding of the emergency scene, which will not only make it easy for plan making, but also helpful for an emergency rescue.

\subsection{Display of the total factor of the environment data in an emergency command}

The measurable real image based on MMS technology provides a full street visualization platform for the emergency command. The emergency command center can conduct the command and display a pre-arranged plan in real-image environments.

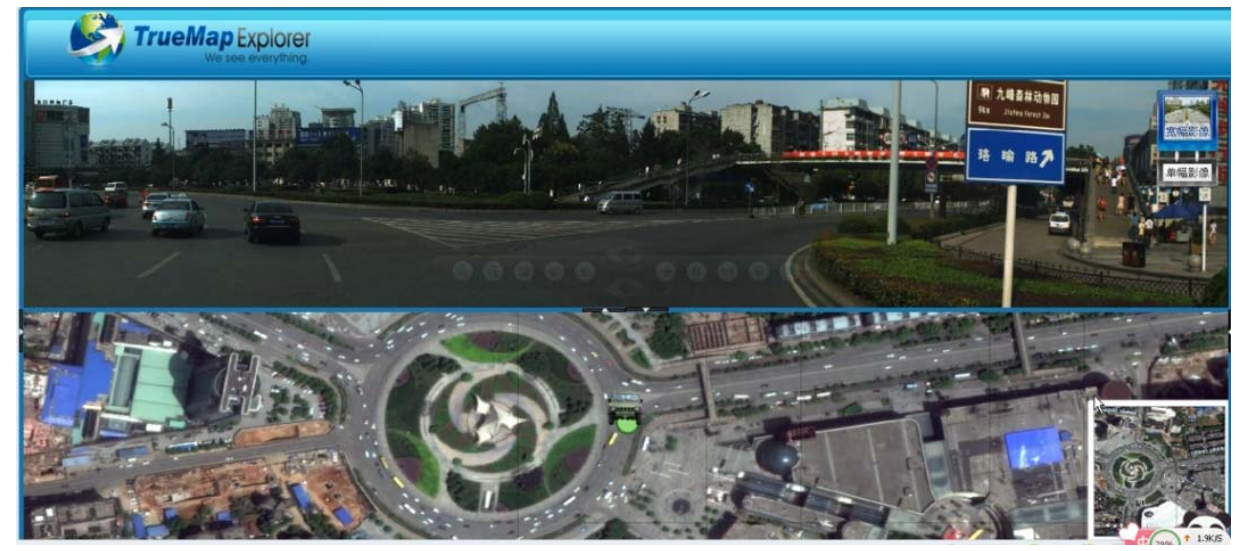

Fig. 4. Display of the total factor of the environment data in an emergency Command

\subsection{Query, mapping and statistics of the visualization emergency facility}

The query function is mainly focused on the key targets and emergency facility elements, such as fire hydrants, fire brigades, etc. For a fire hydrant, you can obtain the information about its properties, such as ground/underground, water/no water, new/old style, pressure conditions and so on. For key targets, the name, address, telephone, number of floors in the main building, the main building height and other information can be obtained. For emergency rescue teams, you can get information like the number of personnel, the number of vehicles, etc. Of course, you can also check the information of the general objectives and get their category and name information. 
The users can enter some particular or a few properties of the device in the query criteria field for simple or compound queries:

1. Implements digital management on the construction projects and urban public emergency facilities, for instance, the user can enquire all the high-rise buildings located at the Renmin Road, or display the image in the format of thematic maps or full street view. Compound query, for example, to search all the municipal fire hydrants located within $500 \mathrm{~m}$ range around the department store; Or to query if there are oil and gas service stations within $50 \mathrm{~m}$ range around a highrise building, and as well as to query all the oil stations with more than 50 tons of reserves on the Renmin Road.

2. Conducts disaster risk assessment of the region. For example, by quantifying the fire risk of each individual building, to analyze and assess the fire risk of the entire region, thus to identify the fire-risk areas and carry out specific fire drills, additional fire-fighting facilities to eliminate the fire risks and ensure fire safety as much as possible.

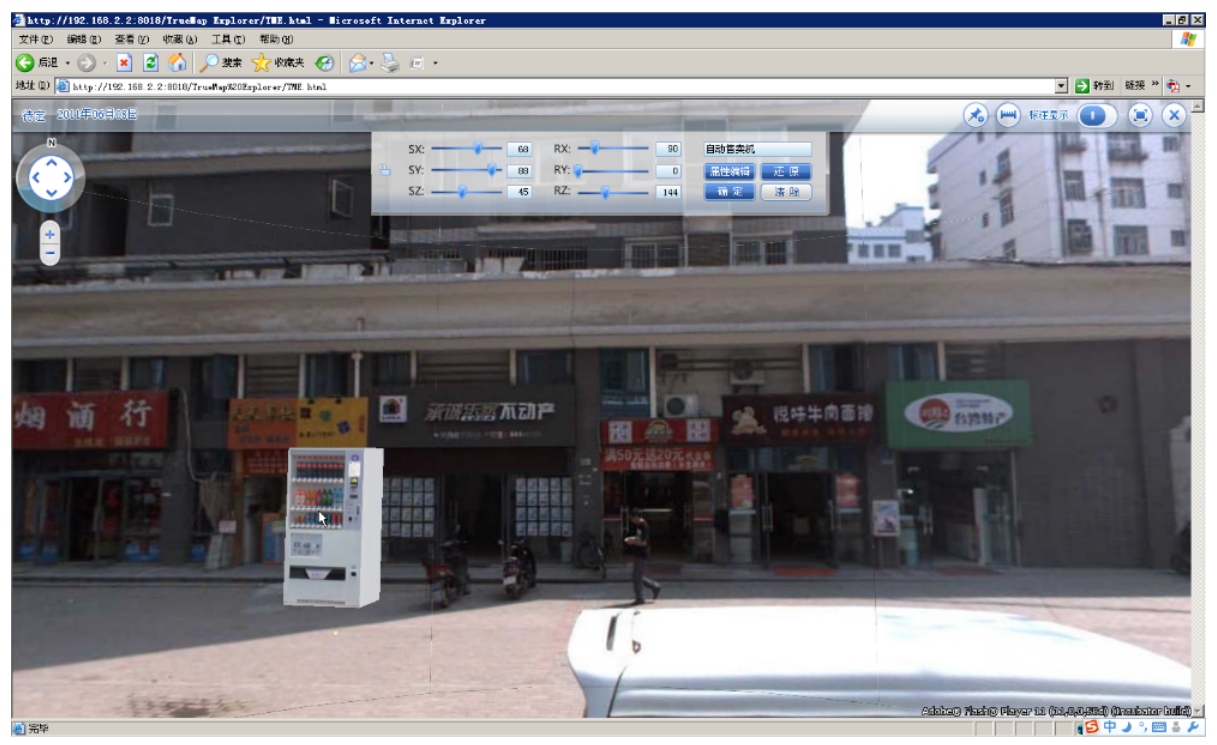

Fig. 5. Infrastructure query and analysis

\subsection{Plan making and drill}

Clicking on the key objectives on the electronic map, you can directly obtain the target objects and the detailed real image of its surrounding within one meter range. This function allows the police officers to develop a field view of the key buildings and its surrounding environment, therefore helps them to better develop contingency plans. In a way, the "TRUEMAP real map" provides comprehensive, multi-angle, measurable real geographic data, which is like the "electronic sand table". Making plan models with the help of the TRUEMAP can greatly improve the accuracy of plan-making and also facilitate the routine drills and field training. 


\subsection{Decision support}

Compared to the traditional two-dimensional vector graphics, "TRUEMAP real map" can display details of the city in a more comprehensive, accurate, intuitive way; therefore it is the best choice for the emergency system basic geographic data. Its main advantages are:

1. Contains rich amount of information which is absent in the traditional maps, such as environment, resources, population, economic information, so that it is more conducive to the operational control of the emergency response;

2. The emergency system involves collaboration and interaction between multiple departments. Since the current map formats and standards used in different departments are various, which results in the fact that the geographic information cannot be shared for use in an emergency response. While the image map has visual uniqueness by using real images to display the objects, its open data management is able to support database integration with any application systems and existing databases, which makes it possible to establish a unified geographic referential standard between the various emergency sub-systems;

3. Get rid of using abstract symbols in the display of the information, instead of using a user-friendly way to convey environmental information, thus to reduce the map-reading time and the mistakes. It is the "electronic sand table" in the computer, which allows the users to obtain valuable information from the images in the emergency command, such as the vehicle traffic width, position of the escape window, the road scene along the emergency lanes, etc.

4. Good vision at night and under bad weather conditions, so that the environment status can still be obtained clearly.

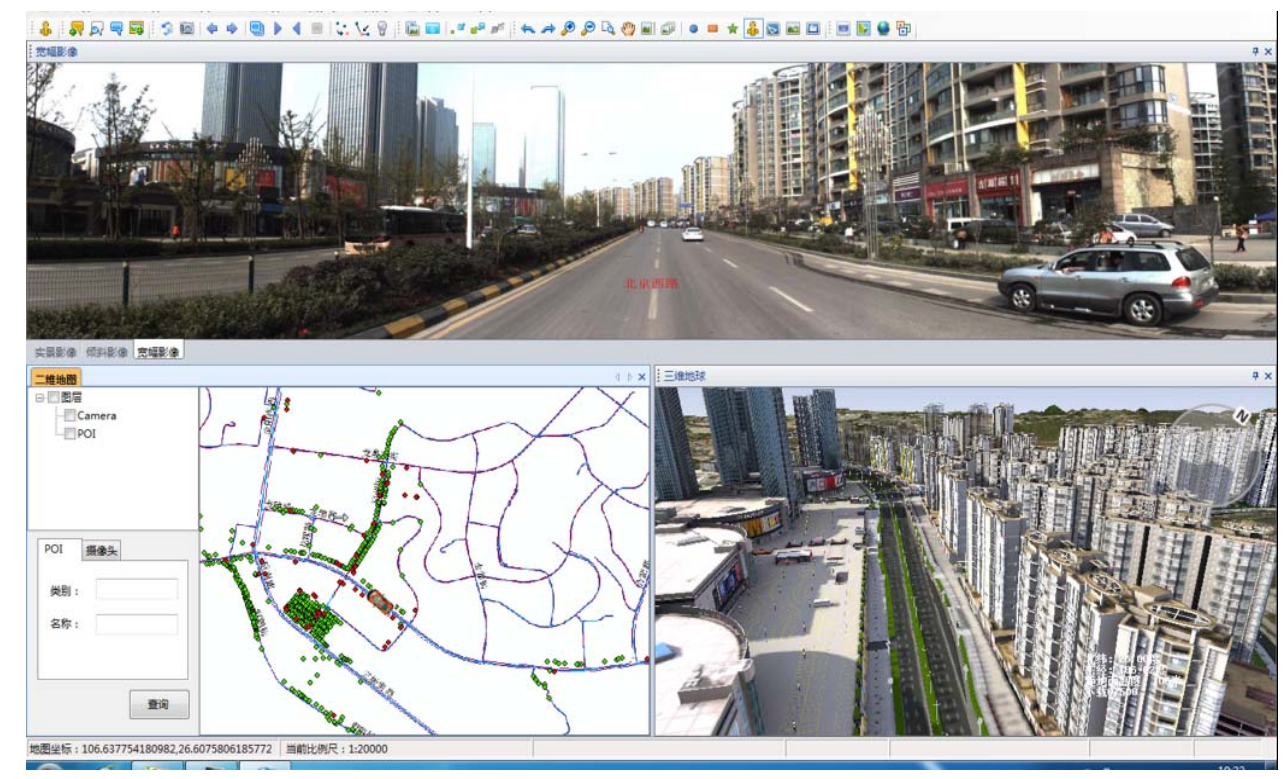

Fig. 6. TRUEMAP diagram 


\section{Conclusion}

The technical characteristic of a Mobile Mapping Systems (MMS) is that it can efficiently integrate and coordinate various technologies, such as GPS, INS, CCD, GIS, computer technology and automatic control technology together. It thus makes the road data collection tasks to be totally relied on in manual collection in a safe, high-quality and efficient way, and also to improve greatly the efficiency of data processing in industry. MMS is a cutting-edge technology integrating a variety of high-tech technologies.

This study of mobile mapping and its key technologies not only provides valuable real three-dimensional geographic information for the government, enterprises and the public, but also offers an opportunity for urban management business, location-based service demands and real three-dimensional images to integrate. In this way real image 3D visualization application environment is developed, that provides a complete solution based on real three-dimensional GIS applications.

Acknowledgements: This work was supported by the Special Scientific Research Fund of Surveying,Mapping and Geoinformation Public Welfare Profession of China (201512026), and the Open Foundation of Key laboratory of watershed ecology and geographical environment monitoring, National Administration of Surveying, Mapping and Geoinformation of China (WE2015016).

\section{References}

1. The Pioneer. USA National Geospatial-Intelligence Agency, May-June 2004.

2. Z hu, Q ing. A Survey of Three-Dimensional Geographic Information System Technologies. Geographic Information World, Vol. 3, 2004, No 3, pp. 8-12.

3. B o, $\mathrm{H} \mathrm{u} \mathrm{a} \mathrm{j} \mathrm{i} \mathrm{e} \mathrm{Three-Dimensional} \mathrm{Abstract} \mathrm{and} \mathrm{Expression} \mathrm{of} \mathrm{Urban} \mathrm{Surface} \mathrm{Landscape.} \mathrm{Chinese}$ Academy of Surveying and Mapping, 2006.

4. Y o n g hu i, Xi a o. Three-Dimensional Modelling and Visualization of Urban Landscape. The PLA Information Engineering University, 2006.

5. W a n g, X., C. L i, Y. Z h o u. Multi-Sensor Infromation Fusion Technology. - Journal of Beijing University of Aeronautics and Astronautics, 2004, No 4.

6. L i, D., S. G u o, Q. H u. 3S (RS, GPS, GIS) Integration Technology Based LD2000 Series Mobile Mapping System and Its Applications. - Acta Geodaetica et Cartographica Sinica, 2008, No 3.

7. L i, D. On Concept and Application of Digital Measurable Images - from 4D Production to 5D Production. - Science of Mapping and Surveying, 2007, No 4.

8. L i, D., Q. Hu. Spatial Information Service Based on Digital Measurable Images. - Geomatics and Information Science of Wuhan University, 2007, No 5.

9. Y i, Y., H. Hu a n g. Components and Application of Vehicle-Borne Mobile Mapping System. Heilong Jiang Science and Technology of Water Conservancy, 2011, No 3.

10. Y u, Z., Y i Y a n g, Z. G u. Application of Mobile Road Mapping System in Road Inforamtion Construction. - Shan Xi Architecture, 2012, No 5.

11. Che n, J. Directions of GIS Development in China: An Overview. - Geomatics World, 2003, No 1.

12. W h i t e, P. D., R. R. J o n e s. A Cost-Efficient Solution to True Color Terrestrial Laser Scanning. - Geosphere, Vol. 4, 2008, No 3, pp. 564-575.

13. B o s c h, J. A. Coordinate Measuring Machines and Systems. Edited by Marcel Dekker. New York, 1995. 
14. Kang, Jinsuk. A Study on the Geographic Recognition for Handheld Computers and Smartphones in Location-Based Services. - In: Proc. of 2012 International Conference on Software and Computer Applications (ICSCA’2012).

15. Research on Laser Range Scanning and Its Application. - Geospatial Information Science, Vol. 4, 2001, No 1, pp. 37-42.

16. Wang, G., G. Chen, Li Yan, H. Chen. The Study of Digital Dynamic Information Management System for Maize Based on Webgis [A]; Progress of Information Technology in Agriculture. - In: Proc. of 4th International Symposium on Intelligent Information Technology in Agriculture (ISIITA), Information Technology Institute, Jilin Agriculture University, 2007.

17. C a o, H., G. Che n, J. Hou. Dept of Mechatronics Engineering. North China University of Science and Technology, Taiyuan 030051, China; Researching Ballistic Location Method Using GPS [A]. - In: Proc. of 5th International Symposium on Test and Measurement, Vol. 1, [C], 2003.

18. Design and Optimization of Query System Architecture Based on Multitier Metadata. [A]. In: Proc. of 27th Session of the Chinese Control Conference, 2008. 